\title{
Experiment Teaching Case Design for Practice Teaching of Photoelectric Sensor Signal Processing Base on CIS Filtering
}

\author{
Yaohua Deng, Jiayuan Chen, Liming Wu, Hui Chen \\ School of Information Engineering of Guangdong University of Technology, \\ Guangzhou Guangdong China \\ dengyaohua@21cn.com
}

Keywords: Experiment Teaching, Photoelectric Sensor Teaching, Contact Image Sensor, FIR

\begin{abstract}
In this paper, one experiment teaching case design for photoelectric sensor signal processing is proposed, by using the filtering of contact image sensor (CIS) as a example. The signal with the noise of CIS the scanning output signal will affect the authenticity of the image, so based on the analysis of the CIS output signal noise, the design of two-dimensional wavelet transform for the reconstruction of the image by the CIS output signal of the filter which is based on Xilinx FPGA dsp48 structurally and realized the accelerated calculation through the FIR IPCore, will be introduced in this paper. From the experiment, this algorithm can reduce most of the noise in reconstructed image to ensure reconstructed image can be complete and clear.
\end{abstract}

\section{Introduction}

At present, the requirements of modern production and scientific research on image acquisition system are increasing day by day. The traditional image acquisition speed slow, its processing function is simple, using discrete components and the circuit is very complex; Furthermore, with poor reliability and not easy to debug, it can not good enough to meet the special requirements; The noise largely affects the images collected so that the scanning image is not so clear and can not to rebuild the image completely [1]. This paper is the CIS sensor signal acquisition based on FPGA (field programmable gate array) which is a special integrated circuit in one of the highest degree. In order to achieve the logic function which is the user desired, the users can reconfigure the logic module and the I/O module of the FPGA inside [2-3]. The single circuit system with many different circuit functions can meet the requirements of different types of CIS sensor interface and achieve the CIS sensors of different types of plug-and-play expediently, which can improve the compatibility of acquisition module and reduce the cost of hardware development again, so it has application value in the field of CIS image scanning.

\section{CIS FPGA Hardware Algorithm for Signal Filtering}

CIS is a CMOS image sensor, including MOS transistor and MOS capacitor element. From the device point of view, the noise of the MOS capacitor is mainly performed by the array spatial noise produced by the manufacturing uneven. MOS transistor, used in most of the CIS, is the main noise source device. The one hand, the noise of MOS transistor is inherent in the device, on the other hand, caused by the circuit structure and working mode. The noise in the output signal of CIS is mainly KTC, $1 / f$, low frequency noise and broadband white noise. So, the main noise in the CIS will be analyzed.

In the process of the CIS acquisition, we often introduce some random noise which will have a certain impact on the quality of the image, and affect the accuracy of the algorithm for Image Mosaic and Target Detection. This paper uses the method of wavelet transform filter which can keep the clear outline of the original image when it removes the image noise to eliminate random noise.

The filtering algorithm used in this paper process is as follows: firstly, the image signal collected by CIS wavelet transform, secondly, to get the specific sub-pictures from wavelet decomposing and 
mean filter. The low frequency sub-band from the image doesn't mean filtering processing, only the high frequency sub-band processing, which can keep the outline of the image better. Based on each scale of the high frequency sub-band image, we can mean filter to achieve the de-noising. From the wavelet decomposition, a sub-image, $\mathrm{H} 1$, of the image after the row low-pass and column high-pass filtering contains the image signal which is in the horizontal direction of the high frequency information and in the vertical direction of the low frequency information. Taking the horizontal alignment template shown in the Figure1 (a) by mean filtering, can not only remove the noise in the horizontal direction, but also retain the low frequency information in the vertical direction; A sub-image, V1, of the image after the column low-pass and row high-pass filtering contains the image signal which is in the vertical direction of the high frequency information and in the horizontal direction of the low frequency information. Taking the vertical alignment template shown in the Figure1 (b) by mean filtering, can not only remove the noise in the vertical direction, but also retain the low frequency information in the horizontal direction; A sub-image, D1, of the image after the row high-pass and column high-pass filtering contains the image signal which is in the horizontal and vertical direction of the high frequency information, which is the diagonal direction of the high frequency information. Figure 1(c) shows the $\mathrm{X}$ shape template filtering. After inverse wavelet transform, we can get the images de-noising from all the sub-band images [4].

$$
\frac{1}{3}\left[\begin{array}{lll}
1 & 1^{*} & 1
\end{array}\right] \frac{1}{5}\left[\begin{array}{lllll}
1 & 1 & 1^{*} & 1 & 1
\end{array}\right] \quad \frac{1}{3}\left[\begin{array}{c}
1 \\
1^{*} \\
1
\end{array}\right] \frac{1}{5}\left[\begin{array}{c}
1 \\
1 \\
1^{*} \\
1 \\
1
\end{array}\right] \quad \frac{1}{5}\left[\begin{array}{ccc}
1 & 0 & 1 \\
0 & 1^{*} & 0 \\
1 & 0 & 1
\end{array}\right]\left[\begin{array}{cccccc}
1 & 0 & 0 & 0 & 1 \\
0 & 1 & 0 & 1 & 0 \\
9 & 0 & 1^{*} & 0 & 0 \\
0 & 1 & 0 & 1 & 0 \\
1 & 0 & 0 & 0 & 0
\end{array}\right]
$$

Fig.1.Mean filter template

In recent years, the wavelet transform is in the attached great importance to new technology in image processing, which is for image compression, feature detection and many new methods of texture analysis like resolution analysis, time-frequency domain analysis, pyramid algorithm and so on, which

are all ultimately attributable to the scope of the wavelet transform. All the small wavelets are carried out from the basic wavelets through the scale expansion and displacement. With special properties, basic wavelet is a real-valued function which is shock attenuation and usually decays very quickly [5]. In mathematics, it meets the conditions of the integral zero:

$$
\int_{-\infty}^{\infty} \psi(t) d t=0
$$

And its spectrum meets the conditions:

$$
C_{\psi}=\int_{-\infty}^{\infty} \frac{|\psi(s)|^{2}}{s} d s<\infty
$$

In the field of digital image processing, wavelet transform generally appears in discrete form. The general form of the discrete wavelet transform is as follows:

$$
\left(W_{\psi}, f\right)(m, n)=<f, \psi_{m, n}>=\left|a_{0}\right|^{\frac{m}{2}} \int_{R} f(x) \psi\left(\frac{\left(x-n a_{0}^{m} b_{0}\right)}{a_{0}^{m}}\right) d x
$$

Among them, $\psi_{m, n}(x)=a_{0}^{\frac{n}{2}} \psi\left(\frac{x-n b_{0}}{a_{0}^{n}}\right)$ called discrete small base wave, in addition, $m, n \in Z$ and $a_{0}, b_{0}$ are the two constants, generally, $a_{0}>0, b_{0}>0$.

One of the most common digital filters is a linear time-invariant (LTI) filter. A LTI response depends on its input signal through a process called linear convolution. Linear convolution defined as $y=f^{*} x$, among them, $\mathrm{f}$ for filter impact response, $\mathrm{x}$ for its input signal and $\mathrm{y}$ for the convolution results. Linear convolution formal process defined for:

$$
y[n]=x[n] * f[n]=\sum_{k} x[k] f[n-k]=\sum_{k} f[k] x[n-k]
$$

LTI digital filter can be divided into finite impulse response (FIR) filter and infinite impulse response (IIR) filter. FIR filter is taking the sum of the limited sampling signal convolution as the 
output of the filter while IIR filter is taking the unlimited one. A constant coefficient FIR is a LTI digital filter. Let the length of the FIR filter for L, then (according to the formula (4)), FIR can be defined as:

$$
y[n]=x[n] * f[n]=\sum_{k=0}^{L-1} f[k] x[n-k]
$$

Corresponding to the FIR impulse response, its Z-transform is for:

$Y[z]=X[z] F[z]$

Among, $F[z]$ is the transmission function for FIR and its Z-transform can be defined as:

$$
F[z]=\sum_{k=0}^{L-1} f[k] z^{-k}
$$

Thus, the structure of the LTI FIR filter length for L according to the formula (7) can be as shown in Figure 3. Known from Figure3, the FIR filter is made up of the tapped delay line, the adder and the multiplier. A parameter in each of the multipliers called the tapped weights is the coefficient of FIR. With the tapped delay line structure, this kind of filter is called the transversal filter.

After turning the input and output of the transversal filter shown in Figure 2, changing the direction of data stream, changing the original adder to a branch point and changing the branch to adder, we can get a new filter structure. Because of transposing from the transversal filter, this kind of filter is called transposed filter and its structure is shown in Figure 3.

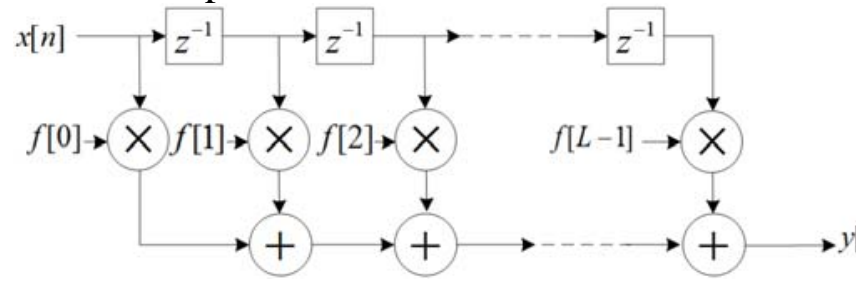

Fig.2.Transversal filter structure diagram

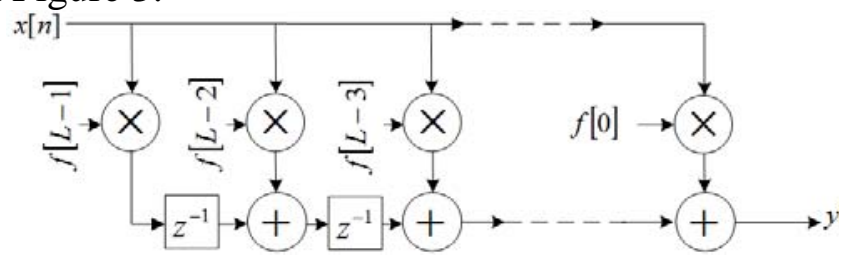

Fig.3.Transposed filter structure diagram

The filter after Transposing is exactly the same as the original filter in the function. From Figure3 and Figure4, it is known that the transposed structure is more suitable for hardware implementation and is helpful to enhance the basic frequency and to reduce the hardware resources. (Because the transversal filter rear-end multistage adder will become the bottleneck of the whole filter processing frequency, we must add more register in front in order to realize synchronous when it adds register.)

\section{Effect test in hardware filtering of CIS output signal to noise}

Data storage is based on the vertical direction of the image to storage shown in the figure, while the black indicates the data storing the pixel position. At the end of every wavelet transform phase, the arrangement distribution of the image pixel is based on the figure above. Now we take the wavelet transform test in the image shown in Figure 4 to contrast the filtering effect.

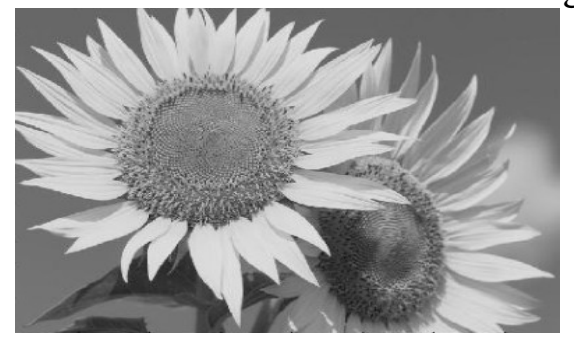

Fig.4.Wavelet transform testing diagram

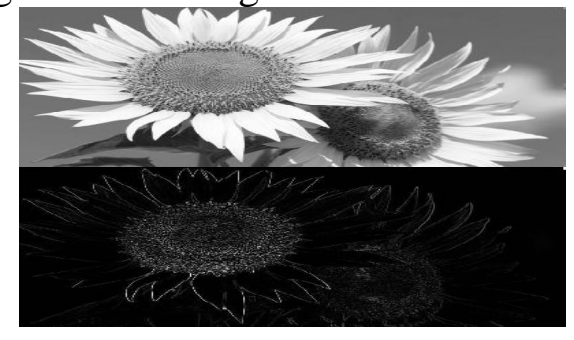

Fig.5.Primary wavelet column transform

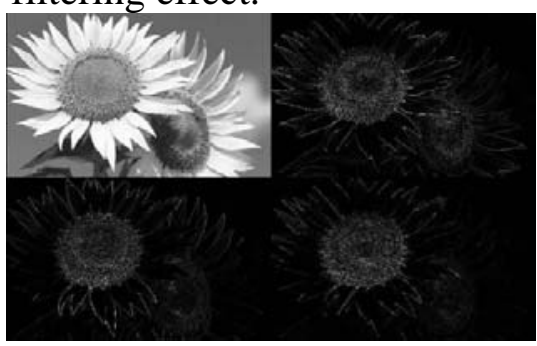

Fig.6.Primary wavelet row transform

The first stage is the vertical direction of primary wavelet transform, retaining the vertical direction of the low-frequency component in the upper part of the image and the high-frequency component in the lower part of the image, as shown in Figure 5.

The second stage is the horizontal direction of primary wavelet transform, retaining the horizontal direction of the low-frequency component in the left half of the image and the high-frequency component in the right half of the image, as shown in Figure 6.

The third stage is the horizontal direction of second wavelet transform, retaining the horizontal 
direction of the low-frequency component in the left half of the image and the high-frequency component in the right half of the image, as shown in Figure 7.

The forth stage is the vertical direction of second wavelet transform, retaining the horizontal direction of the low-frequency component in the left half of the image and the high-frequency component in the right half of the image, as shown in Figure 8.

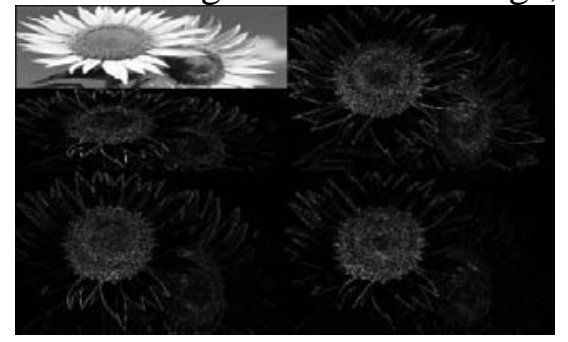

Fig.7.Second wavelet column transform

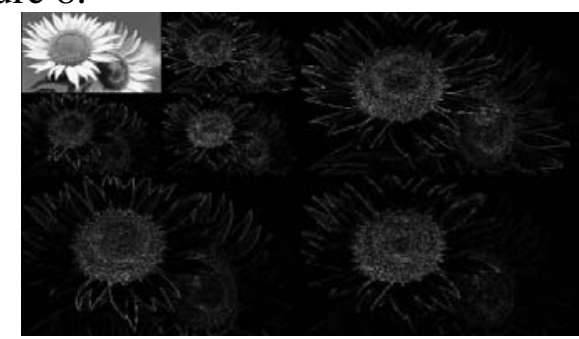

Fig.8.The result of second wavelet row transform

\section{Conclusion}

One effective method for accelerating decomposition or reconstruction of processing image wavelet transform FIR filter is designed in this paper. Using FIR filter to design the decomposition of wavelet operator, to reconstruct and calculate the IP core. The two levels of wavelet decomposition and reconstruction of the IP core will reduce the total consuming time obviously, so the influence of the image noise will be restrained effectively and the scanning image is reconstructed veritably so that the image acquisition will be completely and clearly, providing an initial basis for practice teaching of photoelectric sensor signal processing.

\section{Acknowledgement}

This work is partially supported by Combination project of Industry-university research of Huizhou city of Guangdong Province China (2011C010002008) and Education Teaching Reform project of Guangdong University of Technology (2013ZY008).Thanks for the help.

\section{References}

[1] Y.H. Deng, L.M. Wu, L.K. Zhang. Design of Dual DDS Arbitrary Wave Generator Based on FPGA and Denoising of Spur Noise. Chinese Journal of Scientific Instrument, 2009(11):2255-2261.

[2] B. Zhang, W.K. Yang, L. Xu, et al. Design and Implementation of Arbitrary Waveform Generator Based on FPGA. China Academic Journal Electronic, 2009, 35(1):40-46.

[3] L.M. Wu, J.X. Liu, M. Dai. Single Chip Fuzzy Control System Based on Mixed-Signal FPGA. International Conference on Intelligent Human-Machine Systems and Cybernetics, 2009:397-400.

[4] Y.H. Deng, G.X. Liu, L.M. Wu. Adaptive focus algorithm of image sampling by lifting scheme denoising. Journal of Shanghai Jiaotong University. 2008(12):127-131.

[5] K. Maleknejad, M. Yousefi, K. Nouri.Computational methods for integrals involving functions and Daubechies wavelets. Applied Mathematics and Computation, 2007 (189):1828-1840. 